\title{
PROPORCIÓN CORPORAL, OBESIDAD GENERALY ABDOMINAL EN ADOLESCENTES DE CÓRDOBA, ARGENTINA
}

\section{BODY PROPORTIONS, GENERAL AND ABDOMINAL OBESITY IN ADOLESCENTS FROM CÓRDOBA, ARGENTINA}

\author{
Santiago Rodríguez Lopez ${ }^{1 *}$ y Juan Manuel Bajo² \\ ${ }^{1}$ Centro de Investigaciones y Estudios sobre Cultura y Sociedad (CIECS, CONICET, UNC). Córdoba. Argentina
${ }^{2}$ Facultad de Ciencias Exactas, Físicas y Naturales. Universidad Nacional de Córdoba. Córdoba. Argentina
}

PALABRAS CLAVE índice córmico, perímetro de cintura, índice de cintura-talla, estado nutricional

RESUMEN Se analizó la prevalencia de obesidad general y abdominal (OA) en adolescentes escolares, con diferente proporción corporal, de la ciudad de Córdoba, Argentina. Este es un estudio transversal en el que se incluyeron 614 adolescentes (11-18 años, edad media 13,9 2 2,1; 51,8\% niñas). Se obtuvieron datos de tres establecimientos secundarios públicos de la ciudad de Córdoba durante los años 2007-2014. La proporción corporal se estimó mediante el índice córmico ( $\mathrm{IC}=$ talla sentado/talla total $\mathrm{x} 100$ ), y se obtuvieron valores $\mathrm{Z}$ estandarizados según sexo y edad, y cuartiles $\left(\mathrm{Q}_{1-4}\right)$ de estos valores. Los niveles de sobrepeso y obesidad general se calcularon en base al índice de masa corporal $\left[\mathrm{IMC}=\operatorname{peso}(\mathrm{kg}) / \operatorname{talla}(\mathrm{m})^{2}\right]$, siguiendo las referencias de la Organización Mundial de la Salud, y se obtuvieron valores $\mathrm{Z}$ por sexo y edad. La OA se determinó median- te el índice cintura-talla (ICT=perímetro de la cintura/talla); se definió OA cuando ICT $\geq 0,5$. En general, se observó que, comparados con individuos con IC menor, aquellos con IC más altos (con piernas relativamente más cortas) presentan valores $\mathrm{Z}$ medios mayores de IMC e ICT, junto con una mayor prevalencia de sobrepeso, obesidad general y OA. Este patrón fue estadísticamente significativo en las niñas, mientras que entre los niños también se observó, aunque menos marcadamente y sin alcanzar una significación estadística. Considerando los efectos adversos de la obesidad en la salud inmediata y en el futuro de los adolescentes, los individuos con niveles elevados de IC deberían ser especialmente monitoreados en estudios nutricionales y de crecimiento. Rev Arg Antrop Biol 21(1), 2019. doi:10.17139/ raab.2019.0021.01.06

\section{KEY WORDS sitting-height ratio, waist circumference, waist-to-height ratio, nutritional status}

ABSTRACT We analyzed the prevalence of general and abdominal obesity (AO) in school adolescents with different body proportions, from the city of Córdoba, Argentina. This is a cross-sectional study which included 614 adolescents (11-18 years old, mean age 13,9 $\pm 2,1,51.8 \%$ girls). Data were obtained from three public high schools of Córdoba during the period 2007-2014. Body proportion was estimated via the sitting-height ratio ( $\mathrm{SHR}=$ sitting height/total height $x$ 100), obtaining standardized sex- and age-specific $\mathrm{Z}$ scores, and quartiles $\left(\mathrm{Q}_{1-4}\right)$ of these values. Overweight and obesity were calculated from the body mass index $\left(\mathrm{BMI}=\right.$ weight $(\mathrm{kg}) /$ height $\left.(\mathrm{m})^{2}\right]$, and were defined according to the references provided by the World Health Organization, obtaining sex- and age-specific $\mathrm{Z}$ scores. AO was de-

La utilización de herramientas adecuadas para evaluar el crecimiento, desarrollo, y estado nutricional de niños/as y adolescentes es muy importante para la determinación de su salud inmediata y su bienestar en etapas posteriores del desarrollo. En este sentido, la valoración de las proporciones corporales representa un buen indicador de los procesos de crecimiento y desarrollo mencionados. La alteración de estas proporciones se da mayormente por la competencia entre los distintos segmentos (por ejemplo, tronco versus extremidades inferiores), por es- termined using the waist-to-height ratio $(\mathrm{WHtR}=$ waist circumference / height); AO was established when WHtR $\geq 0.5$. In general, it was observed that, compared with individuals with lower SHR, those with higher SHR (with relatively shorter legs) have higher mean BMI and WHtR Z scores, together with a higher prevalence of overweight, general obesity and AO. While this pattern is statistically significant in girls, it is also found in boys, although less markedly and without reaching statistical significance. Considering the adverse effects of obesity on the current and future health of adolescents, individuals with high levels of SHR should be given special attention in nutritional and growth studies. Rev Arg Antrop Biol 21(1), 2019. doi:10.17139/ raab.2019.0021.01.06

casez de nutrientes durante el embarazo y/o los primeros años de vida, y/o por malas condiciones de salud (Bogin, 2001). Asimismo, durante

Financiamiento: Sin financiamiento.

*Correspondencia a: Santiago Rodríguez Lopez. Centro de Investigaciones y Estudios sobre Cultura y Sociedad. Av. de Valparaíso s/n, Ciudad Universitaria. 5000 Córdoba. Argentina. E-mail: santiago.rodriguez@conicet.gov.ar

Recibido 13 Diciembre 2017; aceptado 1 Mayo 2018

doi:10.17139/raab.2019.0021.01.06 
la infancia y la adolescencia la longitud de las piernas aumenta más rápido que el crecimiento del tronco (Frisancho, 2007). Si en estas etapas críticas de aceleración del crecimiento existen malas condiciones ambientales o los recursos no son suficientes, pueden alcanzarse proporciones antropométricas anómalas, las cuales serán un reflejo de condiciones adversas en etapas tempranas de la vida (Velázquez-Meléndez, Silveira, Allencastro-Souza y Kac, 2005). De esta forma, evaluar las proporciones corporales permite advertir los cambios ontogenéticos que ocurren en ellas, los cuales dependen de una multiplicidad de factores (genéticos, sociales, ambientales, etc.) (Bajo y Mangeaud, 2010).

Por otro lado, la existencia de proporciones antropométricas inadecuadas se asocia a una mayor probabilidad de presentar factores de riesgo cardiovascular en niños y adolescentes, incluida la obesidad (Gasparini Marcato et al., 2010; Molina, Faria, Montero, Cade y Mill, 2010; Rao y Kanade, 2007). De esta manera, se ha evidenciado que aquellos niños/as y adolescentes con retraso en el crecimiento y/o con dimensiones corporales alteradas, son más propensos a tener sobrepeso y obesidad (Pliakas y McCarthy, 2010; Popkin, Richards y Montiero, 1996). Esta asociación entre proporción corporal e IMC (índice de masa corporal) ha sido previamente estudiada (Bogin y Beydoun, 2007; Zhang, Chu y Zhao, 2016) aunque en menor medida utilizando otros indicadores de obesidad, tales como la obesidad abdominal (OA) (Brannsether, Eide, Roelants, Bjerknes, y Benedikt Juliusson, 2014; Chou y Mao, 2012). Esto es importante ya que se ha resaltado que la $\mathrm{OA}$ en adolescentes, estimada mediante el perímetro de la cintura o el índice cintura-talla (ICT), una herramienta simple, efectiva y práctica para monitorear obesidad y síndrome metabólico en adolescentes (Zhou et al., 2014), se asocia más fuertemente a factores de riesgo metabólicos que otros indicadores de adiposidad general (Savva et al., 2000). Sin embargo, se ha sugerido que el perímetro de la cintura sigue sin utilizarse consistentemente en relevamientos antropométricos (Schröder et al., 2014).

Basados en lo expuesto anteriormente, el objetivo de este estudio es analizar la prevalencia de obesidad general y abdominal en adolescentes escolares con diferentes proporciones corporales (estimadas a través del índice córmico
IC), un indicador de las condiciones ambientales prepuberales) de la ciudad de Córdoba, Argentina. El IC es, a su vez, uno de los parámetros más utilizados en el diagnóstico clínico de trastornos de crecimiento desproporcionados (Zhang, Zhang y Chu, 2015).

\section{MATERIAL Y MÉTODOS}

\section{Muestra}

El estudio es de corte transversal, no aleatorizado. Se incluyeron 614 adolescentes de entre 11 y 18 años (edad media 13,9 92,1 ), de los cuales el 51,8\% son niñas. Los datos fueron obtenidos en tres establecimientos secundarios públicos de la ciudad de Córdoba, Argentina, durante los años 2007-2014: Colegio Nacional de Montserrat (426 individuos, 45,3\% niñas), Instituto Provincial de Enseñanza Media (IPEM) No268 (113 individuos, 65,5\% niñas), e IPEM N ${ }^{\circ} 124$ (75 individuos, 68,0\% niñas). La distribución de los individuos por colegio y edad se muestra en la Tabla 1.

El Colegio Nacional de Montserrat es un establecimiento público que depende de la

TABLA 1. Distribución de la muestra por establecimientos escolares y edad

\begin{tabular}{ccccc}
\hline \multicolumn{5}{c}{ Niños (n=296) Niñas (n=318) } \\
& $\mathrm{n}$ & $\%$ & $\mathrm{n}$ & $\%$ \\
\hline Colegio & & & & \\
\hline C.N. de Montserrat & 233 & 78,7 & 193 & 60,7 \\
IPEM N $^{\mathrm{o}}$ 124 & 24 & 8,1 & 51 & 16,0 \\
IPEM N $^{\circ}$ 268 & 39 & 13,2 & 74 & 23,3 \\
\hline Edad (años) & & & & \\
\hline 11 & 50 & 16,9 & 25 & 7,9 \\
12 & 62 & 20,9 & 71 & 22,3 \\
13 & 45 & 15,2 & 46 & 14,5 \\
14 & 45 & 15,2 & 52 & 16,4 \\
15 & 24 & 8,1 & 36 & 11,3 \\
16 & 32 & 10,8 & 39 & 12,3 \\
17 & 29 & 9,8 & 33 & 10,4 \\
18 & 9 & 3,0 & 16 & 5,0 \\
\hline
\end{tabular}

C.N.:Colegio Nacional; IPEM:Instituto Provincial de Enseñanza Media. 
Universidad Nacional de Córdoba (Bajo y Mangeaud, 2010), mientras que el IPEM Nº268 es un colegio urbano ubicado en el barrio céntrico de Nueva Córdoba. A diferencia de los anteriores, el IPEM $\mathrm{N}^{\circ} 124$ es un establecimiento educativo definido como urbano marginal, ubicado al sureste hacia la periferia de la ciudad, y al que concurren también alumnos de zonas rurales aledañas. Queda emplazado en una zona que incluye a barrios precarios y asentamientos marginales cercanos a zonas rurales (Ruderman, Navarro, Mangeaud, Cejas y Bajo, 2017).

La evaluación antropométrica a los adolescentes se realizó luego de la devolución de un consentimiento informado por parte de los padres/madres. Solo fueron incluidos en el estudio aquellos alumnos/as cuyos padres, o tutores legales (o ellos mismos en el caso de ser mayores de edad), firmaron el consentimiento informado. El estudio fue realizado siguiendo las recomendaciones del Protocolo de Helsinki $(1975 / 1983 / 2008)$ y respetando la Ley Nacional 25.326 sobre Privacidad de los Datos. Previo a la realización del estudio se obtuvieron las autorizaciones del Comité de Bioética de la provincia de Córdoba, C.I.E.I.S del Polo Sanitario Sur y de los/as directores/as de los establecimientos educativos.

\section{Variables antropométricas}

El relevamiento antropométrico se realizó siguiendo los procedimientos estandarizados recomendados por el Anthropometric Standardization Reference Manual (Lohman, Roche y Martorell, 1988). Se obtuvieron las siguientes variables antropométricas directas: talla $(\mathrm{cm})$, talla sentado $(\mathrm{cm})$, peso $(\mathrm{kg})$ y perímetro de cintura $(\mathrm{cm})$. A fin de facilitar la comparación entre individuos, se calcularon además valores estandarizados (valores Z) por sexo y edad para cada una de las variables y para la totalidad de los índices estimados. Los valores $\mathrm{Z}$ para cada indicador, se obtuvieron mediante el siguiente cálculo (González Montero de Espinoza y Marrodán, 2007):

$$
Z=\frac{X-\mu}{\sigma}
$$

donde $X$ es el valor a transformar que presenta cada individuo; $\mu$ representa la media la distri- bución original de cada indicador (para cada edad y sexo); $\boldsymbol{\sigma}$ es la desviación estándar de dicha distribución.

\section{Proporción corporal}

La proporción corporal se estimó mediante el índice córmico $[\mathrm{IC}=($ talla sentado/talla total $) \mathrm{x}$ 100]. Para los análisis se clasificaron a los individuos según cuartiles de valores $Z\left(Q_{1-4}\right)$ de $I C$ por sexo y edad $\left(\mathrm{Q}_{1}: \mathrm{IC}<_{\text {percentil }} 25 ; \mathrm{Q}_{2}\right.$ : ${ }_{\text {percentil }} 25 \leq \mathrm{IC}$ $<_{\text {percentil }} 50 ; \mathrm{Q}_{3}: \quad$ percentil $50 \leq \mathrm{IC} \quad<_{\text {percentil }} 75 ; \mathrm{Q}_{4}$ : IC $\left.\geq_{\text {percentil }} 75\right)$. De esta forma, y en comparación con el resto, los individuos en $\mathrm{Q}_{4}$ (mayor IC) presentan extremidades inferiores relativamente cortas para la talla total.

\section{Obesidad general}

Se estimó el IMC mediante la fórmula peso $(\mathrm{kg}) / \operatorname{talla}(\mathrm{m})^{2}$. Posteriormente, se obtuvieron las categorías nutricionales aplicando los valores de referencia para población infantil y juvenil provistos por la Organización Mundial de la Salud mediante el software AnthroPlus (WHO, 2009), obteniendo valores $\mathrm{Z}$ o desviaciones estándar (DE) por sexo y edad: normopeso (entre $-2 \mathrm{DE}$ y $+1 \mathrm{DE})$, sobrepeso $(>+1 \mathrm{DE})$ y obesidad $(>+2 \mathrm{DE})$.

\section{Obesidad abdominal}

La OA se determinó a través del índice cintura-talla (ICT), al dividir el perímetro de la cintura por la talla total (Schröder et al., 2014). Primero se obtuvieron directamente los valores de perímetro de cintura para todos los individuos, medida en el punto medio entre la última costilla y la cresta ilíaca. Teniendo en cuenta la utilidad del ICT para la identificación de la OA en edad pediátrica (Marrodán et al., 2014), se definió la OA según ICT $\geq 0,5$ (Browning, Hsieh y Ashwell, 2010).

\section{Análisis estadístico}

Se realizaron análisis descriptivos de las variables estudiadas. La asociación entre la proporción corporal y los distintos indicadores de obesidad fue evaluada mediante análisis bivariantes (chi-cuadrado y ANOVA, cumpliéndose todos los supuestos para este último análisis). Todos los análisis fueron realizados con el programa estadístico Stata 14.0. (Stata Corp., 2015). 


\section{RESULTADOS}

La Tabla 2 muestra las características antropométricas de los adolescentes. En cuanto al estado nutricional, no se observaron diferencias estadísticamente significativas entre niños y niñas, aunque la obesidad general pareciera levemente mayor entre los niños ( $8,8 \%$ vs. $4,0 \%$; $p=0,058)$. Sin embargo, existe una mayor prevalencia de OA entre las niñas $(22,5 \%$ vs. $14,0 \%$; $p<0,005$ ) como producto de un mayor ICT (media 0,45 vs. 0,$47 ; p<0,01)$, las cuales presentan, además, valores de IC superiores $(p<0,001)$.

$\mathrm{La}$ asociación entre distintas proporciones corporales (cuartiles $\left(\mathrm{Q}_{1-4}\right)$ de IC) y valores estandarizados de indicadores de obesidad se muestra en la Tabla 3. Se observa una tendencia general en dónde aquellos individuos con IC más altos (piernas relativamente más cortas) presentan valores $\mathrm{Z}$ medios mayores de IMC e ICT. Este patrón es estadísticamente significativo tanto para el IMC como para el ICT en las niñas, mientras que entre los niños lo es solo para el IMC.

La Tabla 4 muestra la prevalencia de las distintas categorías nutricionales y de la $\mathrm{OA}$ en individuos con diferentes proporciones corpora- les. En las niñas, aquellas con IC más elevado $\left(\mathrm{Q}_{4}\right)$ presentan mayor prevalencia de sobrepeso, obesidad, y OA que otras con IC menor $(p<0,05)$. Entre los niños se observa una tendencia a una mayor prevalencia de $\mathrm{OA}$ en los individuos con IC más alto $\left(\mathrm{Q}_{3} \mathrm{y} \mathrm{Q}_{4}\right)$, aunque ésta no es estadísticamente significativa. En el caso del IMC este patrón es menos marcado y no resulta significativo, aunque sí se observa algo más claramente para la obesidad general.

\section{DISCUSIÓN}

Este estudio examina la prevalencia de obesidad general y abdominal en adolescentes con diferente proporción corporal, reflejada en $\mathrm{Q}_{1-4}$ de IC. Los resultados obtenidos sugieren que aquellos adolescentes con mayor IC (con miembros inferiores relativamente más cortos) poseen valores estandarizados según sexo y edad de sobrepeso, obesidad general y abdominal, más elevados que aquellos con IC más bajo, sobre todo en las niñas. Estos resultados coinciden en general con estudios previos que describen la relación entre alto IC y mayor prevalencia de sobrepeso y obesidad general (Rao y Kanade, 2007; Zhang et al., 2016). Dicha asociación es

TABLA 2. Indicadores antropométricos de la muestra

\begin{tabular}{cccccr}
\hline & \multicolumn{2}{c}{ Niños } & \multicolumn{3}{c}{ Niñas } \\
Variables & \multicolumn{2}{c}{$(\mathrm{n}=296)$} & \multicolumn{2}{c}{$(\mathrm{n}=318)$} & $p$ valor* \\
\hline Talla (cm), media (DE) & 161,8 & $(11,8)$ & 157,5 & $(7,6)$ & $>0,001$ \\
Peso (kg), media (DE) & 53,2 & $(13,7)$ & 50,9 & $(11,0)$ & $>0,005$ \\
Talla sentado (cm), media (DE) & 82,4 & $(6,6)$ & 82,0 & $(4,5)$ & 0,630 \\
Perímetro de cintura (cm), media (DE) & 73,3 & $(9,7)$ & 73,4 & $(9,8)$ & 0,820 \\
IC, media (DE) & 50,9 & $(1,5)$ & 52,1 & $(1,5)$ & $>0,001$ \\
IMC (kg/m²), media (DE) & 20,1 & $(3,6)$ & 20,4 & $(3,7)$ & 0,231 \\
\hline Categorías de IMC, n (\%) & & & & & 0,058 \\
Normopeso & 226 & $(76,4)$ & 256 & $(79,8)$ & \\
Sobrepeso & 44 & $(14,9)$ & 52 & $(16,2)$ & \\
Obesidad & 26 & $(8,8)$ & 13 & $(4,0)$ & \\
\hline ICT, media (DE) & 0,45 & $(0,05)$ & 0,47 & $(0,06)$ & $>0,010$ \\
OA, n (\%) & 42 & $(14,0)$ & 73 & $(22,5)$ & $>0,005$ \\
\hline
\end{tabular}

$p$ valor*:correspondiente a pruebas t y análisis de chi-cuadrado para variables continuas y categóricas, respectivamente; IC:índice córmico; IMC:índice de masa corporal; ICT:índice cintura-talla; OA:obesidad abdominal. 
TABLA 3. Valores $Z$ por edad y sexo del índice de masa corporal y del indice cintura-talla en adolescentes con diferente índice córmico

\begin{tabular}{ccccc}
\hline & & & $Z_{\text {IMC }}$ & $Z_{\text {ICT }}$ \\
Sexo & IC & $n$ & \multicolumn{2}{c}{ Media $\pm D E$} \\
\hline Niños & $\mathrm{Q}_{1}$ & 72 & $-0,05 \pm 1,14$ & $-0,14 \pm 0,97$ \\
& $\mathrm{Q}_{2}$ & 73 & $0,23 \pm 1,00$ & $-0,03 \pm 0,94$ \\
& $\mathrm{Q}_{3}$ & 77 & $0,35 \pm 1,11$ & $0,02 \pm 0,92$ \\
& $\mathrm{Q}_{4}$ & 74 & $0,65 \pm 1,11$ & $0,15 \pm 1,12$ \\
& & \multicolumn{3}{c}{$\mathrm{F}=5,129 \mathrm{p}<0,05 \mathrm{~F}=1,139 \mathrm{p}=0,334$} \\
\hline Niñas & $\mathrm{Q}_{1}$ & 82 & $-0,18 \pm 1,10$ & $-0,20 \pm 0,89$ \\
& $\mathrm{Q}_{2}$ & 80 & $0,04 \pm 1,01$ & $-0,08 \pm 1,00$ \\
& $\mathrm{Q}_{3}$ & 78 & $0,17 \pm 0,96$ & $-0,02 \pm 0,87$ \\
& $\mathrm{Q}_{4}$ & 80 & $0,62 \pm 1,07$ & $0,30 \pm 1,13$ \\
& \multicolumn{3}{c}{$\mathrm{F}=8,743 \mathrm{p}<0,01$} & $\mathrm{~F}=3,771 \mathrm{p}<0,05$ \\
\hline
\end{tabular}

IMC:índice de masa corporal; ICT:índice cintura-talla; IC:índice córmico; Q:cuartiles; Q1:IC < p25; Q2:p25 $\leq$ IC $<$ p50; Q3:p50 $\leq \mathrm{IC}<$ p75; Q4:IC $\geq$ p75.

relevante ya que se ha sugerido que la obesidad en niños y adolescentes puede ser un mediador entre el IC y otros factores de riesgo cardiometabólicos (Gasparini Mercato et al., 2014), y que a su vez los individuos con obesidad durante la adolescencia tienen más riesgo de ser obesos en la edad adulta (Gordon-Larsen, The y Adair, 2014).

Por otra parte, se ha sugerido que la proporción corporal influencia el IMC. Es decir, siendo el tronco relativamente más pesado que las piernas, la relación tronco/piernas condiciona el IMC al obtenerse mayores valores de éste en individuos con mayor IC (Galloway, ChateauDegat, Egeland y Young, 2011). Es por ello que además del IMC, resulta interesante analizar otros indicadores de obesidad, tales como la OA. Recientemente se ha descripto la asociación entre el IC y la OA en un gran estudio de adolescentes chinos (Zhang et al., 2015). Al igual que en el caso de obesidad general, nuestros resultados coinciden con este último estudio, donde un IC más elevado se asocia a mayor prevalencia de OA, aunque solo significativamente entre las niñas. Estas diferencias entre niños y niñas podrían explicarse, al menos en parte, por la estimación indirecta de la longitud de la pierna. Esta práctica, aunque ampliamente aceptada puede llevar a un sesgo, especialmente en individuos con niveles de sobrepeso y obesidad (Bogin y Varela-Silva, 2010). Es probable que en nuestro estudio, donde aproximadamente el $15 \%$ tiene sobrepeso y el $6 \%$ obesidad, la acumulación de grasa en los glúteos (sobre todo en las niñas) lleve a un incremento de la talla sen-

TABLA 4. Comparación de la prevalencia de normopeso, sobrepeso, y obesidad, y de obesidad abdominal, entre adolescentes con diferente índice córmico

\begin{tabular}{|c|c|c|c|c|c|}
\hline Sexo & IC & Normopeso & $\begin{array}{c}\text { Sobrepeso } \\
\mathrm{n}(\%)\end{array}$ & Obesidad & $\begin{array}{c}\mathrm{OA} \\
\mathrm{n}(\%)\end{array}$ \\
\hline \multirow[t]{5}{*}{ Niños } & $\mathrm{Q}_{1}$ & $60(83,3)$ & $9(12,5)$ & $3(4,2)$ & $8(11,1)$ \\
\hline & $\mathrm{Q}_{2}$ & $56(76,7)$ & $13(17,8)$ & $4(5,5)$ & $6(8,2)$ \\
\hline & $\mathrm{Q}_{3}$ & $59(76,7)$ & $10(13,0)$ & $8(10,4)$ & $14(18,2)$ \\
\hline & $\mathrm{Q}_{4}$ & $51(68,9)$ & $12(16,2)$ & $11(14,9)$ & $14(18,9)$ \\
\hline & & \multicolumn{3}{|c|}{$\mathrm{Chi}^{2}=7,963 ; p=0,241$} & $\mathrm{Chi}^{2}=5,065 ; p=0,167$ \\
\hline \multirow[t]{4}{*}{ Niñas } & $\mathrm{Q}_{1}$ & $71(86,6)$ & $10(12,2)$ & $1(1,2)$ & $13(16,0)$ \\
\hline & $\mathrm{Q}_{2}$ & $67(83,8)$ & $10(12,5)$ & $3(3,8)$ & $13(16,3)$ \\
\hline & $\mathrm{Q}_{3}$ & $63(80,8)$ & $13(16,7)$ & $2(2,6)$ & $18(23,1)$ \\
\hline & $\mathrm{Q}_{4}$ & $54(67,5)$ & $19(23,8)$ & $7(8,8)$ & $27(34,2)$ \\
\hline
\end{tabular}


tado, que en definitiva incidirá en el incremento del IC (Bogin y Varela-Silva, 2008).

La tendencia y evolución hacia la obesidad infantil y adolescente debe ser fuertemente monitoreada (Cole, 2010). En los últimos 10-20 años la adiposidad central ha aumentado dramáticamente en los adolescentes (Rivera et al., 2014), incluso en mayor medida que la adiposidad general (McCarthy y Ashwell, 2006). Se ha señalado que la Argentina es uno de los países latinoamericanos con mayor sobrepeso y obesidad (Lomaglio, 2012). Estudios nutricionales en adolescentes en nuestro país señalan una prevalencia de sobrepeso y obesidad de $28,6 \%$ y 5,9\%, respectivamente (Dirección de Promoción de la Salud y Control de Enfermedades No Transmisibles, 2013), existiendo variaciones regionales y un gradiente norte-sur en exceso de peso (sobrepeso y obesidad) (Oyhenart et al., 2008). Específicamente en Córdoba, un estudio reciente en adolescentes escolares reportó valores de 13\% (sobrepeso) y $8 \%$ (obesidad) (Andreo y Salcedo Corallo, 2016). El sobrepeso y obesidad en nuestro estudio son elevados y similares a los reportados previamente, aunque las prevalencias varían en función de las muestras analizadas, las herramientas para el cálculo del estado nutricional, etc. En nuestro estudio al igual que en éste último, no se registraron individuos con bajo peso. La ausencia o poca incidencia de bajo peso, junto con la existencia de sobrepeso y obesidad, es reflejo de lo que ocurre unas muchas regiones de nuestro país, donde poblaciones adolescentes están transitando un proceso de transición nutricional avanzado.

Otros estudios relacionan la valoración de la proporción corporal con la presencia de diversos indicadores de desnutrición (Bergel Sanchís et al., 2016). Un aspecto interesante de nuestro estudio, al igual que en muchos otros, es que examina la asociación entre el IC y distintos indicadores de obesidad en adolescentes escolares. Sin embargo, también presenta algunas limitaciones que es necesario resaltar para interpretar correctamente los resultados obtenidos. Primero, si bien se cuenta con información sobre el contexto socioeconómico general de los alumnos/as, brindada por los propios establecimientos educativos, no fue posible la obtención de información completa sobre el contexto socioeconómico de la familia de cada adolescente. Esto último no es una restricción menor, ya que el hecho de no poder estimar el nivel socioeconómico de los individuos impide poder establecer la asociación entre éste, la propia historia nutricional y/o de salud, y el IC de los adolescentes. Es decir, al no contar con esta información debemos asumir lo que históricamente sugiere la literatura (Leitch, 1951); esto es, que aquellos individuos provenientes de entornos socioeconómicamente más desfavorecidos poseen IC más alto (con extremidades inferiores relativamente más cortas). Estudios con poblaciones no patológicas indican que el medio ambiente influye en mayor medida sobre la longitud de las piernas y las proporciones corporales que, por ejemplo, los genes (Bogin y Varela-Silva,2010). En otras palabras, suponemos que el IC es un indicador válido del entorno socioeconómico y nutricional en el cual han crecido los individuos. Sin embargo, otros autores sugieren que lo anterior se observa más claramente en las poblaciones occidentales, pero que no debe asumirse como regla general en aquellos países cuyas primeras generaciones están experimentando la transición epidemiológica y el desarrollo económico asociado (Schooling et al., 2008a; 2008b). Luego, tampoco contamos con información completa sobre los patrones de dieta y actividad física a nivel individual, lo que también limita el estudio. Además, tampoco han sido incluidos otros indicadores de crecimiento puberal o de maduración, los cuales podrían influenciar el IC.

Finalmente, al ser un estudio no aleatorizado, los resultados obtenidos no pueden extrapolarse a la población de adolescentes escolares de Córdoba, por lo que lo evidenciado en este estudio debe ser interpretado dentro de las limitaciones expuestas.

\section{CONCLUSIONES}

En resumen, valores altos de IC que reflejan extremidades inferiores relativamente cortas, se asocian a mayor sobrepeso, obesidad, y OA entre las niñas. Considerando los efectos adversos de la obesidad en la salud inmediata y en el futuro de los adolescentes, los individuos con niveles de desproporción corporal (reflejado en un elevado IC) deberían ser especialmente monitoreados en estudios nutricionales y de crecimiento. 


\section{AGRADECIMIENTOS}

Un agradecimiento especial de los autores hacia las autoridades, padres/madres, y alumnos/ as de los tres colegios relevados en este estudio.

\section{LITERATURA CITADA}

Andreo, M. J. y Salcedo Corallo, A. 2016. Validación del uso de segmentos corporales para valorar el peso y talla de niños/as y adolescentes de la ciudad de Córdoba. Tesina de licenciatura. Facultad de Ciencias Médicas, Universidad Nacional de Córdoba, Córdoba.

Bajo, J. M. y Mangeaud, A. 2010. Relationship between the lung function and anthropometric measures and indexes in adolescents from Córdoba, Argentina. American Journal of Human Biology, 22,823-829. doi:10.1002/ ajhb. 21090

Bergel Sanchís, M., Quintero, F., Navazo, B., Cesani, M., Garraza, M., Torres, M., Luna, M., Luis, M., Castro, L. y Oyhenart, E. 2016. Caracterización del estado nutricional en relación con factores socio-ambientales de la población escolar del partido de Punta Indio (Provincia de Buenos Aires, Argentina). Revista Argentina de Antropología Biológica, 18(2),1-13. doi:10.17139/ raab.2016.0018.02.09

Bogin, B. 2001. The growth of humanity. New York, United States: Willey Liss.

Bogin, B. y Beydoun, N. 2007. The relationship of sitting height ratio to body mass index and fatness in the United States, 1988-1994. Human Ecology, 15:1-8.

Bogin, B. y Varela-Silva M. I. 2008. Fatness biases the use of estimated leg length as an epidemiological marker for adults in the NHANES III sample. International Journal of Epidemiology, 37(1),201-209. doi:10.1093/ije/dym254

Bogin, B. y Varela-Silva M. I. 2010. Leg length, body proportion, and health: A review with a note on beauty. International Journal of Environmental Research and Public Health, 7,1047-1075. doi:10.3390/ijerph7031047

Brannsether, B., Eide, G., Roelants, M., Bjerknes, R. y Benedikt Juliusson, P. 2014. Interrelationships between anthropometric variables and overweight in childhood and adolescence. American Journal of Human Biology, 26(4),502-510. doi:10.1002/ajhb.22554

Browning, L. M., Hsieh, S. D. y Ashwell, M. 2010. A systematic review of waist-to-height ratio as a screening tool for the prediction of cardiovascular disease and diabetes: 0.5 could be a suitable global boundary value. $\mathrm{Nu}$ trition Research Reviews, 23(2),247-269. doi:10.1017/ S0954422410000144

Chou, C. y Mao, Z. 2012. Waist-to-height ratio and obesity in chinese. En V. Preedy. (Ed.), Handbook of Anthropometry: Physical Measures of Human Form in Health and Disease (pp. 2007-2015). New York, United States: Springer.

Cole, T. J. 2000. Secular trends in growth. Proceedings of the Nutrition Society, 59,317-324. doi:10.1016/S1570677X(02)00033-3

Dirección de Promoción de la Salud y Control de Enfermedades No Transmisibles. 2013. $2^{\circ}$ Encuesta Mundial de Salud Escolar Argentina 2012. Ministerio de Salud de la Nación. Argentina. Recuperado de: https://www. msal.gob.ar/ent/images/stories/vigilancia/pdf/2013-0301_guias-evaluacion.pdf

Frisancho, A. 2007. Relative leg length as a biological marker to trace the developmental history of individuals and populations: Growth delay and increased body fat. American Journal of Human Biology, 19(5),703-710. doi:10.1002/ajhb.20676

Galloway, T., Chateau-Degat, M-L., Egeland, G. E. e Young T. K. 2011. Does sitting height ratio affect estimates of obesity prevalence among Canadian Inuit? Results from the 2007-2008 Inuit Health Survey. American Journal of Human Biology, 23,655-663. doi:10.1002/ajhb.21194

Gasparini Marcato, D., Dutra Sampaio, J., Roberty Badiani Alves, E., Silva Araujo de Jesus, J., Teixeira Bessa Fuly, J., Bermudes Giovaninni, N. y Fiorot Costalonga, E. 2014. Sitting-height measures are related to body mass index and blood pressure levels in children. Arquivos Brasileiros de Endocrinologia \& Metabologia, 58,802-806. doi:10.1590/0004-2730000003312

González Montero de Espinosa, M. y Marrodán, M. D. 2007. La salud escolar también se mide. Guía práctica para docentes. Madrid, España: Ed. Centro Regional de Innovación y Formación Las Acacias.

Gordon-Larsen, P., The, N. y Adair, L. 2011. Longitudinal trends in obesity in the US from adolescence to the third decade of life Obesity, 18(9),1801-1804. doi:10.1038/ oby. 2009.451

Leitch, I. 1951. Growth and health. British Journal of Nutrition, 5, 142-151.

Lohman, T., Roche, A. y Martorell, R. 1988. Anthropometric Standardization Reference Manual. Champaign, United States: Human Kinetics Publishers.

Lomaglio, D. 2012. Transición nutricional y el impacto sobre el crecimiento y la composición corporal en el noroeste argentino (NOA). Nutrición Clínica y Dietética Hospitalaria, 32(3),30-35.

Marrodán, M. D., Martínez Álvarez, J., González-Montero de Espinosa, M., López-Ejeda, N., Cabañas, M. D, Pacheco, J. L., Mesa, M. S., Prado, C. y Carmenate, M. M. 2011. Estimación de la adiposidad a partir del índice de cintura talla: Ecuaciones de predicción aplicables en población infantil española. Nutrición Clínica y Dietética Hospitalaria, 31:45-51.

McCarthy, H. y Ashwell, M. 2006. A study of central fatness using waist-to-height ratios in UK children and adolescents over two decades supports the simple message 'keep your waist circumference to less than half your height'. International Journal of Obesity, 30(6),988992. doi:10.1038/sj.ijo.0803226

Molina, M. dC., Faria, C. Pd., Montero, M. P., Cade, N. V. y Mill, J. G. 2010. Cardiovascular risk factors in 7-to-10year-old children in Vitória, Espírito Santo state, Brazil. Cadernos de Saúde Pública, 26,909-917. doi:10.1590/ S0102-311X2010000500013

Oyhenart, E., Dahinten, S., Alba, J., Alfaro, E., Bejarano, I., Cabrera, G. E., Cesani, M. F., Dipierri, J., Forte, L., Lomaglio, D., Luis, M., Luna, M., Marrodán, M., Moreno Romero S., Orden, O., Quintero, F., Sicre, M., Torres, M., Verón, J. y Zavatti J. 2008. Estado nutricional infanto juvenil en seis provincias de Argentina: Variación regional. Revista Argentina de Antropología Biológica, 10(1):1-62.

Pliakas, T. y McCarthy, H. 2010. Association of leg length with overweight and obesity in children aged 5-15 years: A cross-sectional study. Annals of Human Biology, 37(1),10-22. doi:10.3109/03014460903092371

Popkin, B., Richards, M. y Montiero, C. 1996. Stunting is associated with overweight in children of four nations that are undergoing the nutrition transition. The Journal of Nutrition, 126,3009-3016.

Rao, S. y Kanade, A. 2007. Somatic disproportion predicts risk of high blood pressure among adolescent 
girls in India. Journal of Hypertension, 25,2383-2389. doi:10.1097/HJH.0b013e3282efff8e

Rivera, J., González de Cossío, T., Pedraza, L., Aburto, T., Sánchez, T. y Martorell, R. 2014. Childhood and adolescent overweight and obesity in Latin America: A systematic review. The Lancet Diabetes \& Endocrinology, 2,321-332. doi:10.1016/S2213-8587(13)70173-6

Ruderman, A., Navarro, T., Mangeaud, A., Cejas, V. y Bajo, J. M. 2017. Somatotipos de adolescentes escolarizados de Córdoba (Argentina). Revista Argentina de Antropología Biológica, 19(2),1-11. doi:10.17139/ raab.2017.0019.02.05

Savva, S., Tornaritis, M., Savva, M. E., Kourides, Y., Panagi, A., Silikiotou, N., Georgiou, C. y Kafatos, A. 2000. Waist circumference and waist-to-height ratio are better predictors of cardiovascular disease risk factors in children than body mass index. International Journal of Obesity Related Metabolic Disorders, 24,1453-1458.

Schooling, C. M., Jiang, C. Q., Heys, M., Zhang, W. S., Adab, P., Cheng, K. K., Lam, T. H. y Leung GM. 2008a. Are height and leg length universal markers of childhood conditions? The Guangzhou Biobank Cohort Study. Journal of Epidemiology and Community Health, 62(7):607-614. doi:10.1136/jech.2007.065003

Schooling, C. M., Jiang, C. Q., Heys, M., Zhang, W. S., Lao, X. Q., Adab, P., Cowling, B. J., Thomas, G. N., Cheng, K. K., Lam, T. H. y Leung, G. M. 2008b. Is leg length a biomarker of childhood conditions in older Chinese women? The Guangzhou Biobank cohort study. Journal of Epidemiology and Community Health, 62(2),160-166. doi:10.1136/jech.2006.058917

Schröder, H., Ribas, L., Koebnick, C., Funtikova, A., Gomez,
S. F., Fíto, M., Perez-Rodrigo, C. y Serra-Majem, L. 2014. Prevalence of abdominal obesity in Spanish children and adolescents. Do we need waist circumference measurements in pediatric practice? PLOS ONE, 9(1),16. doi:10.1371/journal.pone.0087549

StataCorp. (2015). Stata statistical software: Release 14. College Station, TX: StataCorp LP.

Velásquez-Meléndez, G., Silveira, E. A., Allencastro-Souza, P. y Kac, G. 2005. Relationship between sitting-heightto-stature ratio and adiposity in Brazilian women. American Journal of Human Biology, 17(5),646-653. doi:10.1002/ajhb.20423

WHO (2009). WHO AnthroPlus for personal computers Manual: Software for assessing growth of the world's children and adolescents. Geneva. Recuperado de: https://www.who.int/growthref/tools/who_anthroplus manual.pdf

Zhang, Y., Zhang, Z.y Chu, Z. 2015. Prevalence of general and abdominal obesity among children and adolescents with different sitting height ratios in Shandong, China. International Journal of Cardiology, 191,18-19. doi:10.1016/j.ijcard.2015.04.281

Zhang, Y., Chu Z, y Zhao, J. 2016. Distribution of sitting height ratio and its association with body mass index among children and adolescents in Shandong, China. Biology and Medicine, 8,267. doi:10.4172/09748369.1000267

Zhou, D., Yang, M., Yuan, Z., Zhang, D., Liang, L., Wang, C., Zhang, S., Zhu, H., Lai, M. y Zhu, Y. 2014. Waist-to-height ratio: A simple, effective and practical screening tool for childhood obesity and metabolic syndrome. Preventive Medicine, 67(Supplement C),35-40. doi:10.1016/j.ypmed.2014.06.025 